\title{
Retrosigmoid Transmeatal
}

\section{Approach: An Anatomic Study of an Approach Used for Preservation of Hearing in Acoustic Neuroma Surgery and Vestibular Neurotomy}

\begin{abstract}
The limitations of the transmeatal phase of the retrosigmoid transmeatal approach are shown, and the problems of opening the internal acoustic meatus via this approach and the criteria for its use are discussed. (Skull Base Surgery, 3(1):16-21, 1993)
\end{abstract}

Detailed topographic knowledge of the cerebellar surface of the petrous bone, and of the structures lying within the bone, is required when performing acoustic neurinoma surgery and vestibular nerve section. During the restrosigmoid transmeatal removal of an acoustic neurinoma for preservation of hearing, one exposes the internal acoustic meatus posteriorly by drilling away the bone between the endolymphatic sac and posterior semicircular canal posterolaterally and the posterior lip of the internal acoustic porus anteromedially. In this area inner ear structures are hidden within the bone and if damaged would result in loss of hearing. However, exposure of the internal acoustic meatus needs to be made as laterally as possible to facilitate complete removal of a tumor, and there are many different descriptions of the landmarks that can be used for safe exposure of the fundus in the transmeatal approach. ${ }^{1-9}$

This study, based on the dissection of 30 temporal bones, attempts to determine the topographic variations of the internal auditory canal opening and, combined with the senior author's experience of more than 200 cases of removal of acoustic neuromas using the retrosigmoid transmeatal approach, defines the precise indications for the use of this approach in the preservation of hearing.

\section{MATERIAL AND METHOD}

Thirty temporal bones were dissected under microscopic control. Measurements of the distances between the important topographic structures of the cerebellar surface of the petrous bones (Figs. 1,2) were taken by a modified calliper to the nearest $0.5 \mathrm{~mm}$. Having completed the dissections, the topographic relationships between the dissected structures of the inner ear and the internal acoustic meatus were also measured.

After identification of the landmarks on the cerebellar surface of the petrous bone (internal acoustic porus, cochlear canaliculus, endolymphatic sac), the dura mater was cut in a V-shape (Fig. 1) with the base about $2 \mathrm{~mm}$ above and below the porus and joining at the medial edge of the endolymphatic sac. The flap was elevated and

Skull Base Surgery, Volume 3, Number 1, January 1993 ORL Clinic, Safarik University, Kosice, Czechoslovakia, Ear, Nose, and Throat Department, Lewisham Hospital, London, United Kingdom; and O.R.L. Honoraire des Hopitaux de Paris, France Dr. Koval is scholarship holder of the Ministry of Health of France, in Service ORL, Groupe Hospitalier Cochin, Paris, France Reprint requests: Dr. Koval, ORL Clinic, Safarik University, SNP 1, 04011 Kosice, Czechoslovakia Copyright (C) 1993 by Thieme Medical Publishers, Inc., 381 Park Avenue South, New York, NY 


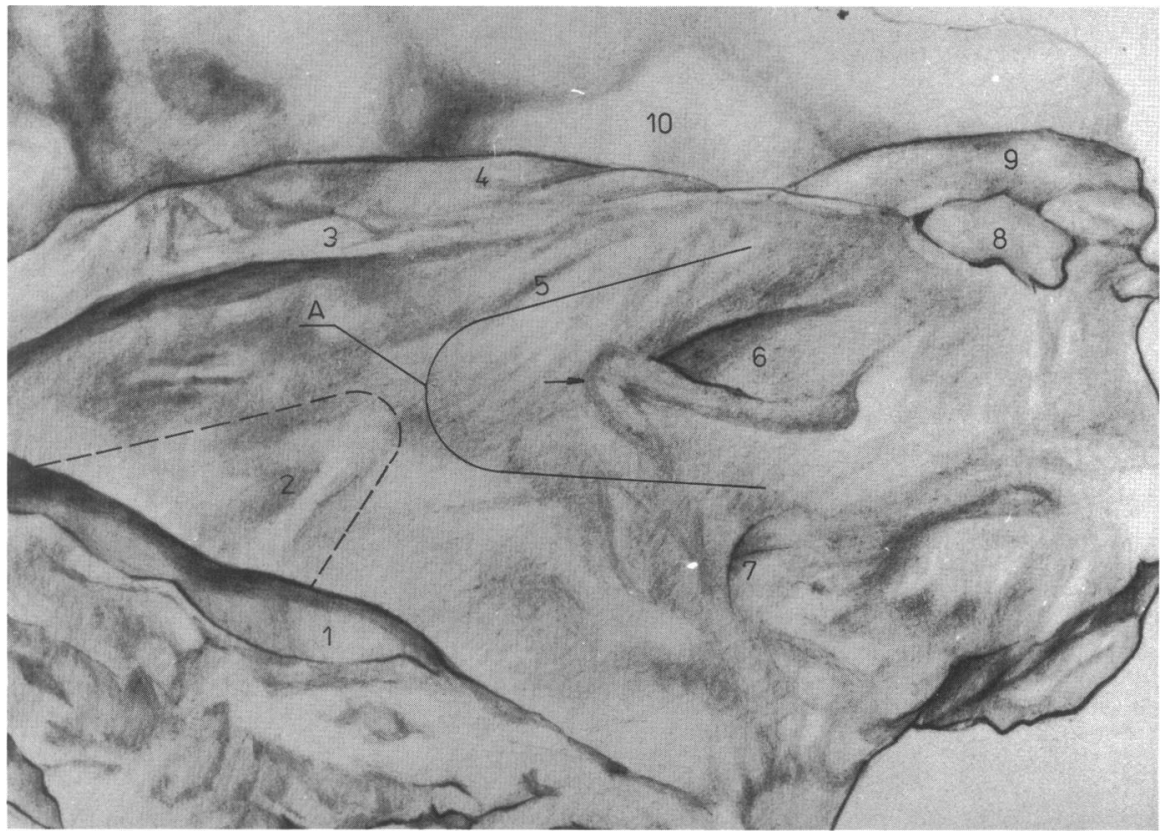

Figure 1. Left temporal bone. Cerebellar side of the pyramid. 1: Sigmoid sinus; 2 : endolymphatic sac; 3: superior petrous sinus; 4: arcuate eminence, 5 : subarcuate fossa; 6 : internal acoustic porus; 7 : cochlear canaliculus; 8: trigeminal nerve; 9: trigeminal ganglion $n . V ; 10$ : middle fossa. A: incision line of the triangular dural flap. Arrow shows the loop of the anterior inferior cerebellar artery.

turned anteromedially. On the exposed bone, the subarcuate fossa was identified with the opening for the subarcuate artery, which was always found within the field. Then, the operculum, the junction of the endolymphatic sac and the vestibular aqueduct, was identified. Its shape varied, although usually it was broad, and sometimes identification was difficult, particularly in well-pneumatized speci- mens. The vestibular aqueduct was then followed to the common crus and the vestibule, which were blue-lined. The vestibular aqueduct is the superolateral limit of the dissection, because the posterior semicircular canal lies immediately lateral to it (Fig. 3).

The lower limit of the dissection is the cochlear canaliculus, and identification of its medial end is usually

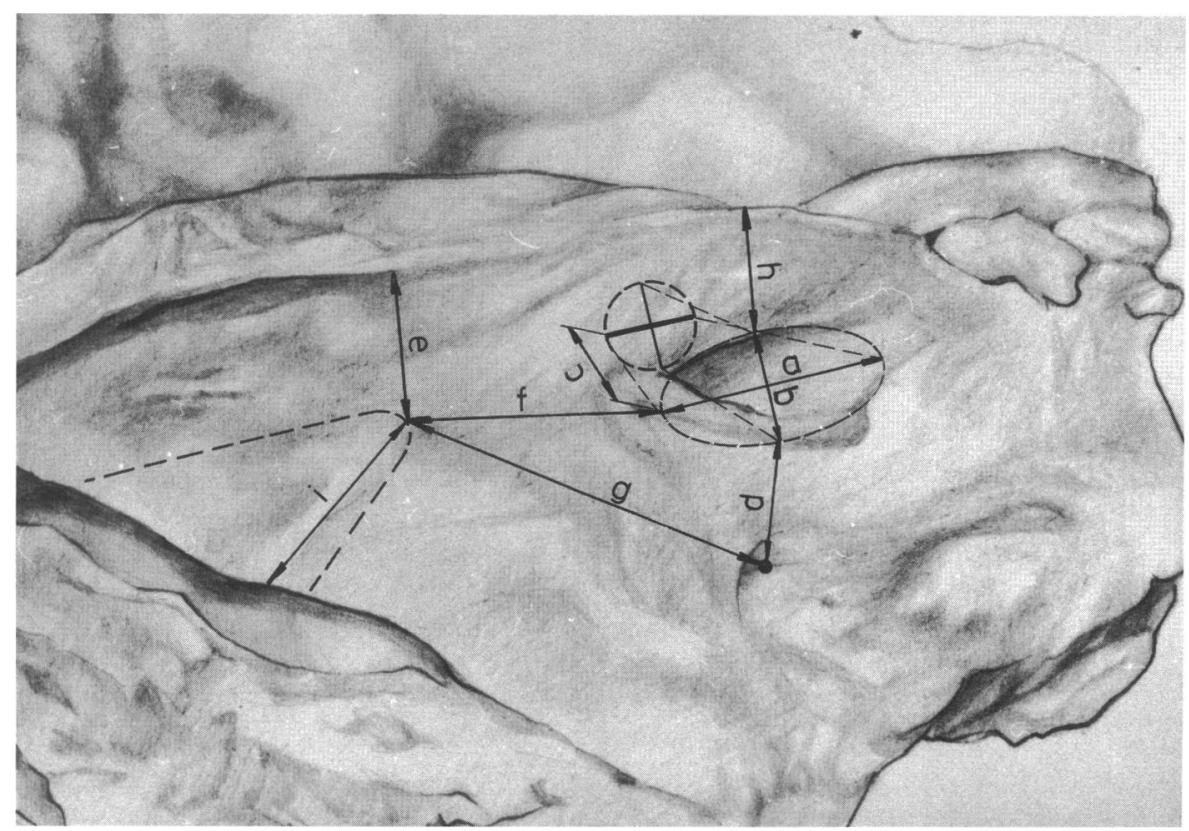

Figure 2. Cerebellar side of the pyramid-measurements. 


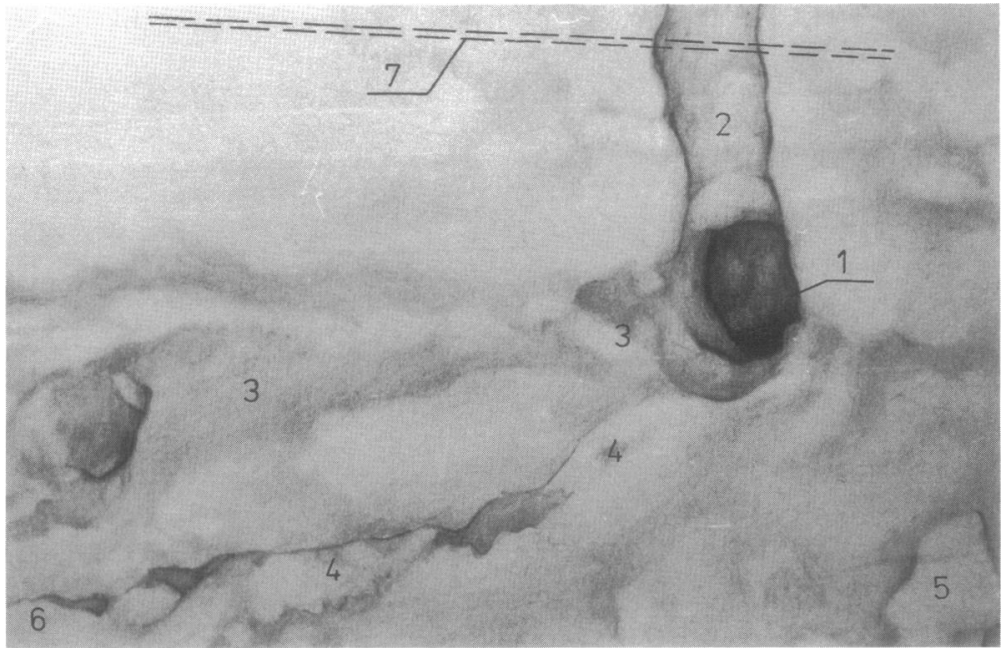

Figure 3. Relationship of the endolymphatic aqueduct and the posterior semicircular canal-detail. 1: Common crus; 2 : superior semicircular canal; 3 : posterior semicircular canal; 4: vestibular aquaeduct; 5: arcuate artery; 6: operculum; 7: superior petrous sinus.

quite easy. The cochlear canaliculus (Fig. 5) was followed laterally on average $5.1 \mathrm{~mm}$ (range, 3.5 to 6), which avoids damage to the posterior semicircular canal ampulla, which was also blue-lined. At almost the same level laterally, the singular nerve was identified. This represented the lower lateral limit of the dissection. The final step was to remove the already thinned bony posterior meatal wall up to the horizontal crest, although a thin bony rim was preserved to facilitate measurements.

On 10 temporal bones, the dissection was carried out with an operating microscope with its optical axis perpendicular to the petrous bone. When dissecting the other 20 bones, the axis was at an angle of $30^{\circ}$ to the petrous bone, thus imitating the operative conditions of the retrosigmoidtransmeatal approach. When dissecting the latter group of bones, we limited the lateral extent of exposure to a level that ensured preservation of the inner ear structures. The posterior meatal wall was removed up to this level and the remaining intact part of the wall was measured. The dissections were then completed under the same limited visual parameters to expose the horizontal crest, and finally the dissection field was reinspected at an angle of $90^{\circ}$.

\section{RESULTS}

The distance between the important topographic structures of the cerebellar side of the petrous bone are shown in Table 1. Of the 10 bones dissected with the microscope in a perpendicular plane, the posterior meatal wall was removed up to the horizontal crest without opening the inner ear. When the optic axis was $30^{\circ}$, only two thirds or so of the posterior meatal wall could be drilled off without damaging the inner ear structures. After completing the dissections at this angle of approach up to the

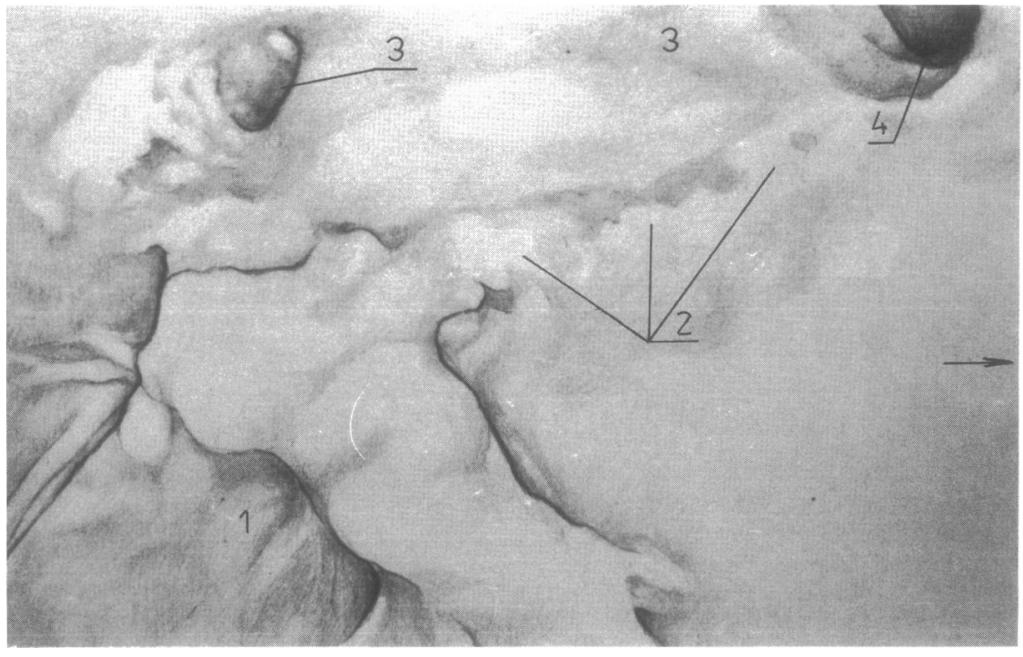

Figure 4. Superior lateral demarcation limit-detail. 1: Endolymphatic sac; 2: endolymphatic duct; 3: posterior semicircular canal; 4: common crus, arrow in the direction of the porus. 
Figure 5. Left cerebellar side of the pyramid, lateral limit of the dissection. 1: Endolymphatic duct; 2: operculum; 3 : singular nerve; 4: cochlear canaliculus; 5: posterior lip of the porus; 6 : dural flap turned to the front; 7 : mixed nerves.

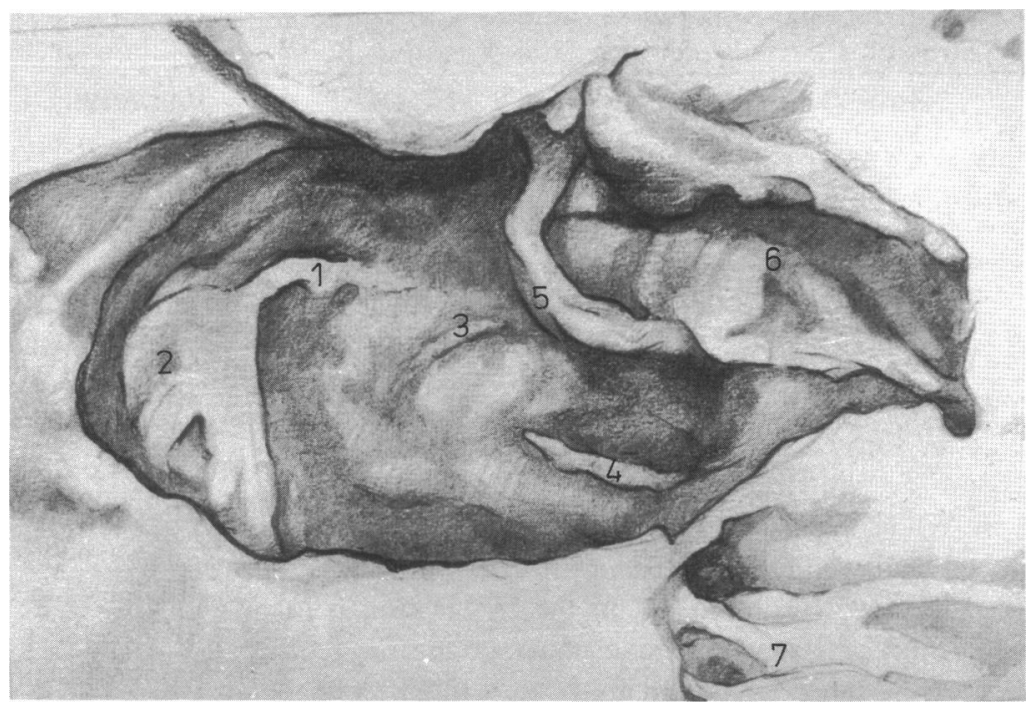

horizontal crest, the inner ear was accidentally opened in 5 of 20 temporal bones.

On all temporal bones, the foramen singulare was found practically at the same lateral level as the posterior semicircular canal ampulla. The horizontal crest was found more medially than the vestibule on all bones. The lamina cribrosa was situated on average $1.5 \mathrm{~mm}$ (range, 1 to 2.5) lateral to the horizontal crest. The horizontal crest is concave in shape and so all measurements were taken from its posterior end. The distance between the jugular bulb and the floor of internal acoustic meatus was, on average, $6.3 \mathrm{~mm}$ (range, 3.5 to 9.5 ). On five bones, the loop of the anterior inferior cerebellar artery was fixed to the dura mater at the posterior lip of the porus (Fig. 1) Table 2 lists the other parameters that were measured. On 10 of 30 temporal bones, the endolymphatic sac was in contact with, or extended beyond, the anteromedial border of the sigmoid sinus.

\section{DISCUSSION}

The cerebellar surface of the petrous bones was inspected prior to dissection (Figs. 1, 2) and its topographic relationships were measured (Table 1). The results were similar to those found by Anson, ${ }^{1}$ Lang, ${ }^{6}$ and Domb and Chole. ${ }^{3}$ In surgery, these parameters are important for demarcating the region of the cerebellar surface of the petrous apex where the dissection is to be performed.

The loop of the anterior cerebellar artery (Fig. 1) was found fixed to the dura mater at the posterior lip of the porus on 5 of 30 temporal bones (16.7\%), Lang6 had found this in only $4 \%$ of specimens.

Of 30 temporal bones, the endolymphatic sac was in contact with (seven cases) or extended beyond the anterior border of the sigmoid sinus (three cases). This finding supports the view that the retrosigmoid approach for a
Figure 6. Posterior meatal wall drilled off up to the horizontal crest. 1: Common crus; 2: region of the arcuate artery; 3 : posterior margin of the meatus; 4 : horizontal crest; 5: superior vestibular nerve compartment; 6: facial nerve compartment; 7: Bill's bar; 8: inferior vestibular nerve compartment; 9: cochlear nerve compartment, 10: inferior vertical crest; 11: middle fossa; 12: superior petrous sinus.

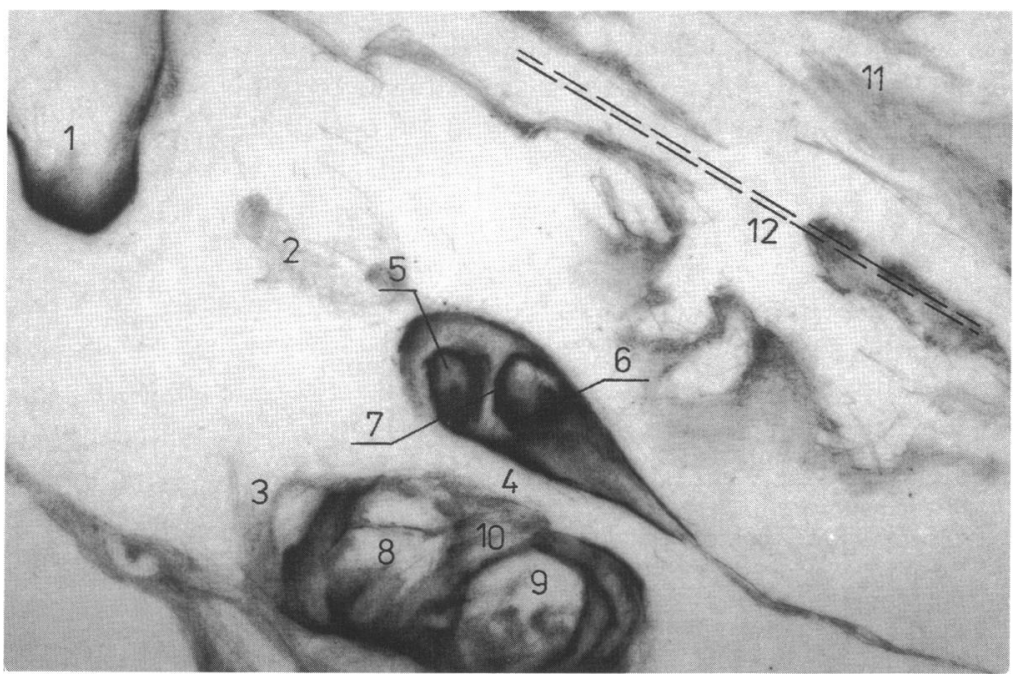


Table 1. Distance Between Important Topographic Structures of the Cerebellar Side of Petrous Bone

\begin{tabular}{lll}
\hline $\mathrm{a}=9.3$ & $(7-12) \mathrm{mm}$ & $\mathrm{f}=11.2(12-8) \mathrm{mm}$ \\
$\mathrm{b}=4.5$ & $(3-6) \mathrm{mm}$ & $\mathrm{g}=13.9(6-10) \mathrm{mm}$ \\
$\mathrm{c}=7.5$ & $(5-10) \mathrm{mm}$ & $\mathrm{h}=5.5(7-4) \mathrm{mm}$ \\
$\mathrm{d}=6.6$ & $(8-5) \mathrm{mm}$ & $\mathrm{i}=12.6(10-15) \mathrm{mm}$ \\
$\mathrm{e}=10.4$ & $(12-8) \mathrm{mm}$ & \\
\hline
\end{tabular}

vestibular neurotomy is safer than the retrolabyrinth approach, since there is no risk of opening the endolymphatic sac, which may cause complete hearing loss. ${ }^{10}$

When the triangular dural flap was freed and the bones were denuded, we found that the opening for the arcuate artery was situated in the trepanation field on all specimens. This artery contributes to the vascularization of the inner ear, and damage to it might contribute to inner ear microcirculatory impairment.

During a retrosigmoid approach, a surgeon can see the cerebellar surface of the petrous apex at an angle of less than $30^{\circ}$. Therefore safety factors limit the trephination field, especially more laterally.

In the laboratory, when dissecting at $90^{\circ}$, it is possible to remove the whole posterior meatal wall up to the horizontal crest without opening the inner ear, because with this orientation, landmarks can be well visualized. If the angle of the view is more acute, one cannot recognize the landmarks as clearly, and the limit of trephination is more medial; thereafter, only a small part of the posterior meatal wall can be drilled off safely without opening the posterior semicircular canal. In our experience, we could only remove approximately two thirds of the posterior meatal wall at an angle of $30^{\circ}$ without damage to the inner ear. If completed up to the horizontal crest, the inner ear was frequently opened.

Silverstein et al9 described a "safe" technique of opening the internal auditory canal but proposed limiting the exposure laterally to the foramen singulare. Guerkirk ${ }^{4}$ recommends following the vestibular aqueduct and the cochlear canaliculus to the point at which they converge, and keeping lateral to that point. In our experience one can rely on these techniques only at an angle of $90^{\circ}$, that is, in the laboratory, which also reveals possible topographic variations of the foramen singulare and the operculum.

Table 2. Measurement of 30 Bones After the Dissections Were Completed*+

\begin{tabular}{llll}
\hline JB-IAM & $6.3(3.5-9.5)$ & IAP-CCr & $11.0(9-13)$ \\
IAP-HC & $7.5(5-10)$ & OP-V & $8.7(6.5-12)$ \\
IAP-V & $8.8(7-11)$ & CCr-HC & $4.5(3.5-5)$ \\
IAP-OP & $13.6(11-16)$ & OP-CCr & $9.1(7.5-10)$
\end{tabular}

*Measurements are in millimeters.

tThe length of the cochlear canaliculus was $5.1(5-6.3)$. FIAM: internal acoustic meatus (bottom); IAP: internal acoustic porus (posterior lip); HC: horizontal crest; V: vestibule; OP: operculum; CCr: common crus; LC: lamina cribrosa; JB: dome of the jugular bulb.
Smith et a ${ }^{2}$ suggest a safe lateral limit of $2 \mathrm{~mm}$ from the horizontal crest, which is close to the limit suggested by our results. Unfortunately, the lateral extent of the fundus in our material was 1 to $2.5 \mathrm{~mm}$ further laterally than the horizontal crest, so that approximately $3 \mathrm{~mm}$ or more of the internal auditory canal remains unexposed.

Roland et al ${ }^{8}$ found a close correlation between measurements taken from computed tomography (CT) scans and dissection specimens of the cerebellar surface of the petrous bone. They suggest that measurements taken from preoperative CT scans may be used as a guide to the lateral aspect of the internal auditory canal and can also demonstrate other topographic variations.

When operating on a patient, the optimal angle of approach to the petrous bone can be obtained by using mannitol $25 \%$ (1 to $15 \mathrm{gm} / \mathrm{kg}$ ) 30 minutes and acetazolamide infusion $(500 \mathrm{mg})$ before incision of the dura mater, or by inserting a lumbar cerebrospinal fluid (CSF) drain. An optimal otoneurosurgical anesthetic is necessary to obtain maximum shrinkage of the cerebellum, thus minimizing the need for retraction. After incision of the retrosigmoid dura mater, the cerebellum is protected by a piece of lyophylized dura mater, the lateral cistern is then opened, and the CSF is evacuated, thus completing the approach to the cerebellopontine angle. ${ }^{11,12}$

The indications for this approach are limited and are affected by the size of the tumor, its relationship to the fundus of the internal auditory canal, and the preoperative hearing level. Sterkers ${ }^{12}$ operated on 180 consecutive cases of acoustic neurinoma by the retrosigmoid approach in an attempt to preserve hearing. The initial percentage with adequate hearing preservation decreased with time to only $20 \%$ of all cases, and in fact only $10 \%$ had a discrimination score greater than $50 \%$ on follow-up. He found that this approach also carried a greater risk of hemorrhage than the translabyrinthine route. For this reason, he added a routine mastoidectomy to the retrosigmoid approach, thus permitting a rapid transformation to the translabyrinthine approach in case of hemorrhage or if the facial nerve is at great risk during dissection of the tumor by the retrosigmoid approach. When limiting the retrosigmoid approach to the removal of tumors that do not invade the meatus, hearing is preserved in $37 \%$ of the cases. Recurrence of the neurinoma can occur if residual tumor is left at the fundus inadvertently.

\section{CONCLUSION}

The transmeatal phase of the restrosigmoid approach for acoustic neuroma removal is difficult because of the limited view of the internal acoustic canal. Training in a temporal bone laboratory is mandatory in order to master the technique of dissection. It should be practiced, taking account of the following points:

1. The cerebellar surface of the petrous bone should be examined full-face in order to become famil- 
iar with the normal anatomy and the variations of the structures on the cerebellar surface side of the petrous bone.

2. The examination should be performed using a limited angle $\left(30^{\circ}\right)$ in order to appreciate the localization of the landmarks before discovering them.

3. Learn how to drill the posterior meatal wall, stopping two thirds along the length of the meatus.

4. Then finish the drilling at $90^{\circ}$ in order to localize the vestibular structures (which should obviously not be opened during operation on a living patient).

\section{REFERENCES}

1. Anson B: Critical distance in the middle and inner ear and posterior cranial fossa. Trans Am Acad Ophtalmol Otolaryngol 76:108129,1972

2. Smith M, Clancy T, Lang J: Consideration of hearing in acoustic neurilemoma excision. Trans Am Acad Ophtalmol Otolaryngol 76:108-129, 1972
3. Domb G, Chole R: Anatomical studies of the posterior petrous apex with regard to hearing preservation in acoustic neuroma removal. Laryngoscope 90:1769-1776, 1980

4. Guerkink N: Surgical anatomy of the temporal bone posterior to the internal auditory canal: An operative approach. Laryngoscope 87:975-986, 1977

5. Kartusch J, Telian S, Graham M, Kemink J: Anatomic basis for labyrinthine preservation during posterior fossa acoustic tumour surgery. Laryngoscope 96:1024-1028, 1986

6. Lang J: Clinical anatomy of the cerebellopontine angle and internal acoustic meatus. Adv Otorhinolaryngol 34:8-24, 1984

7. Rhoton A: The posterior fossa approach to the internal acoustic meatus: Laboratory dissection guide. In Silverstein H, Norrell H (eds): Neurological Surgery of the Ear, volume II. Birmingham AL: Aesculapius Publishing 1979, pp 208-217

8. Roland P, Meyerhoff W, Wright C, Michey B: Anatomic considerations in the posterior approach to the internal auditory canal. Ann Otol Rhinol Laryngol 97:621-625, 1988

9. Silverstein $\mathrm{H}$, Norrell $\mathrm{H}$, Smouha A, Haberkamp T: The singular canal: a valuable landmark in surgery of the internal auditory canal. Otolaryngol Head Neck Surg 98:138-143, 1988

10. Sterkers JM, Renou G: La chirurgie du vertige. G M de France, 83 : 1813-1817, 1976

11. Bouguet D, Brinquin L, Vilotte A, Desgeorges M, Sterkers JM: $L$ 'anesthesie dans la chirurgie des neurinomes de l'acoustique par voies trans-petreuses. Ann Oto-Laryng (Paris) 103:479484, 1986

12. Sterkers JM: Le neurinome de l'acoustique. Les monographies du CCA. Paris: Wagram, 1986, p 97

\section{REVIEWER'S COMMENTS}

The anatomic study by Koval et al is commendable because it further delineates the anatomic relationship of critical structures from a retrosigmoid surgical viewpoint. The findings of this study are helpful to both the novice and the experienced surgeon who use the retrosigmoid approach to resect acoustic neuromas or perform a vestibular neurectomy. As reiterated by their findings, approximately two thirds of the posterior meatal wall can be safely drilled in the surgical position without damage to the inner ear structures. When this drillout is extended to the horizontal crest, a landmark marking the area of safe dissection, the authors encountered damage to the inner ear in 5 of 20 temporal bones. Significant potential hazards for vascular trauma include: (1) fixation of the anteroinferior cerebellar artery to the dura at the posterior lip of the porous acousticus, and (2) the presence of the opening for the arcuate artery within the surgical field.

The authors' commentary on the results of hearing preservation in view of their extensive experience with the retrosigmoid approach reiterates the need for a meticulous understanding of surgical anatomy and proper patient selection. Adequate hearing preservation reported in less than $20 \%$ of 180 consecutive patients with acoustic neuromas undergoing the retrosigmoid approach is disappointing and perhaps related to the tumor's size. Others report more encouraging results with tumors less than $2 \mathrm{~cm}$ in size. ${ }^{1,2}$ Hearing can be preserved in better than $90 \%$ of those having vestibular neurectomies. ${ }^{3}$ Although the authors believe drilling of the internal auditory canal is not necessary for vestibular neurectomy, drilling may indeed be required when no cochlear vestibular cleavage plane exists in the cerebellopontine angle.

13. Harner SG, Beatty CW, Ebersole MJ: Retrosigmoid removal of acoustic neuroma: Experience 1978-1988. Otolaryngol Head Neck Surg 103:40-45, 1990

14. Smith MF, Lagger RL: Hearing a conservation in acoustic neurilemmoma surgery via the retrosigmoid approach Otolaryngol Head Neck Surg 92:168-175, 1984

15. Silverstein $\mathrm{H}$, Norrell $\mathrm{H}$, Haberkamp T. A comparison of retrosigmoid IAC, retrolabyrinthine, and middle fossa vestibular neurectomy for treatment of vertigo. Laryngoscope 97:165-173, 1987

Vinod K. Anand, M.D. 